\title{
Effect on Cavity Optomechanics of the Interaction Between a Cavity Field and a 1D Interacting Bosonic Gas
}

\author{
Qing Sun ${ }^{1,2}$, Xing-Hua $\mathrm{Hu}^{1}$, W. M. Liu ${ }^{1}$, X. C. Xie ${ }^{3,4}$, and An-Chun $\mathrm{Ji}^{2,1}$ \\ ${ }^{1}$ Beijing National Laboratory for Condensed Matter Physics, \\ Institute of Physics, Chinese Academy of Sciences, Beijing 100190, China \\ ${ }^{2}$ Department of Physics, Capital Normal University, Beijing 100048, China \\ ${ }^{3}$ International Center for Quantum Materials, Peking University, Beijing 100871, China and \\ ${ }^{4}$ Department of Physics, Oklahoma State University, Stillwater, Oklahoma 74078, USA
}

(Dated: August 28, 2018)

\begin{abstract}
We investigate the optomechanical coupling between 1D interacting bosons and the electromagnetic field in a high-finesse optical cavity. We show that by tuning the interatomic interactions, one can realize the effective optomechanics with the mechanical resonators ranging from the side-mode excitations of a Bose-Einstein condensate (BEC) to particle-hole excitations of Tonks-Girardeau (TG) gas. We propose that, this unique feature can be formulated to detect the BEC-TG gas crossover and measure the sine-Gordon transition continuously and nondestructively, which are achievable immediately in current experiments.
\end{abstract}

PACS numbers: $37.30 .+\mathrm{i}, 03.75 . \mathrm{Kk}, 42.50 . \mathrm{Pq}$

The experimental achievements in manipulating the strong coupling between ultracold atoms and the electromagnetic field in an optical cavity have triggered many new exciting advances to cavity quantum electrodynamics (QED) [1 $[$ 8]. One of the remarkable achievements is to implement cavity optomechanics with cold atoms [6, 7] or a BEC [8], which is of great importance both for technical applications ranging from optical communication to quantum computation [9], and conceptional exploration of the classic-quantum boundaries [10].

In this Letter, we investigate the optomechanical coupling between a 1D interacting bosonic gas and a cavity field. So far the recent works have neglected the interatomic interactions or considered merely the weakly interacting region, where the mean-field Bogoliubov theory is valid [8, 11]. In this case, the $1 \mathrm{D}$ bosonic gas forms a BEC (or quasi-condensate) 12], and the side-mode excitations of the condensate play the role of mechanical resonator with the bare frequency $\omega_{M}^{0}=4 \hbar k^{2} / M$ 8], here $k=2 \pi / \lambda_{c}$ is the wave-vector of the cavity mode. However, when the interatomic interactions are added into the system, the situation changes dramatically. The strong interatomic interactions would transform the ground state of the condensate to a Luttinger liquid (LL); and remarkably in the strongly interacting limit, the $1 \mathrm{D}$ bosons-known as a TG gas [13 16]-exhibit completely different behavior like ideal fermions. It is therefore important to explore the interatomic interaction effects on the cavity optomechanics, where the quantum fluctuations of 1D bosons are very strong.

In this work, we first employ the quantum hydrodynamical approach to derive an effective model of the cavity QED with 1D interacting bosons. We show that the effective optomechanics can be realized in the intermediately and strongly interacting regions. The corresponding optomechanical coupling is determined by the low-energy excitations or the interatomic interactions of the bosons. Therefore, by probing the cavity oscillations or the noise spectra versus the interatomic interactions, one can determine the quantum phases of the $1 \mathrm{D}$ interacting gas and detect the BEC to TG gas crossover, a fascinating phenomenon of the system [17]. Furthermore, we propose that one could also measure the sineGordon transition, which has stimulated considerable interest [18, 19], conveniently with the nondemolition measurements [20, 21].

The system under investigation is schematically depicted in Fig. 1(a), where $N$ ultracold bosonic atoms of mass $M$ with resonant frequency $\omega_{a}$ are confined in a 1D trap inside an optical cavity with length $L$. The cavity mode of frequency $\omega_{c}$ is driven by a pump laser of frequency $\omega_{p}$ at rate $\eta$, and $\kappa$ is the decay rate of the cavity field. Following Ref. 22], we adiabatically eliminate the internal excited state of the atoms, as justified by the large detuning between the atomic resonance and pump frequency. Then, by using the dipole and rotating-wave approximations, one arrives at the following Hamiltonian of the atomic part

$$
\begin{aligned}
\hat{H}_{a}= & \frac{\hbar^{2}}{2 M} \int_{0}^{L} d x \partial_{x} \hat{\Psi}^{\dagger}(x) \partial_{x} \hat{\Psi}(x)+\int_{0}^{L} d x \hat{V}(x) \hat{\rho}(x) \\
& +\frac{1}{2} \int_{0}^{L} d x d x^{\prime} \hat{\rho}(x) U\left(x-x^{\prime}\right) \hat{\rho}\left(x^{\prime}\right) .
\end{aligned}
$$

Here, $\hat{\Psi}(x)$ is the bosonic field operator and $\hat{V}(x)=$ $\hbar U_{0} \cos ^{2}(k x) \hat{c}^{\dagger} \hat{c}$ is the dynamical periodic potential, with $\hat{c}$ the annihilation operator of a cavity photon, and the potential depth $U_{0}=g_{0}^{2} /\left(\omega_{p}-\omega_{a}\right)$. The interatomic interactions are given by contact pseudo-potentials $U(x-$ $\left.x^{\prime}\right)=g_{1 d} \delta\left(x-x^{\prime}\right)$, where $g_{1 d}=\frac{2 \hbar^{2} a_{s}}{\left(1-\mathcal{C} a_{s} / \sqrt{2} l_{\perp}\right) M l_{\perp}^{2}}$ is the effective 1D coupling strength with $a_{s}$ the threedimensional scattering length, $\mathcal{C}=1.0325$, and $l_{\perp}=$ 


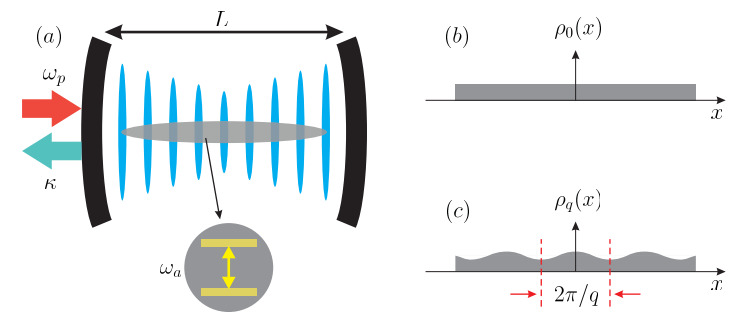

FIG. 1: (color online). Experimental set-up and schematic density distribution. (a) $N$ bosonic atoms with resonant frequency $\omega_{a}$ are confined in an effectively 1D trap inside an optical cavity of length $L$. The cavity mode is driven by a pump laser of frequency $\omega_{p}$, and $\kappa$ is the decay rate. (b) Ground-state atomic density distribution $\rho_{0}(x)$ in the absence of a cavity mode. (c) Distribution of density fluctuation $\rho_{q}(x)$ with wave-vector $q= \pm 2 k$, which is scattered by the periodic potential of the cavity mode.

$\sqrt{\hbar / M \omega_{\perp}}$ the transverse oscillator length.

We start by considering the general situation with arbitrary interatomic interactions and derive an effective model of the the system by using quantum hydrodynamical approach 23], which is a well-defined low-energy theory. We shall work in the low photon numbers limit, where the dynamical periodic potential $\hat{V}(x)$ is feeble. By introducing two new fields $\hat{\phi}(x)$ and $\hat{\theta}(x)$, which describe the collective fluctuations of the phase and density respectively and satisfy the commutation relation $\left[\hat{\phi}(x), \frac{1}{\pi} \partial_{x^{\prime}} \hat{\theta}\left(x^{\prime}\right)\right]=i \delta\left(x-x^{\prime}\right)$, we can express the bosonic field operator $\hat{\Psi}(x)$ as

$$
\hat{\Psi}(x) \sim\left[\rho_{0}-\frac{1}{\pi} \partial_{x} \hat{\theta}(x)\right]^{1 / 2}\left\{\sum_{m=-\infty}^{+\infty} e^{2 m i \hat{\theta}(x)} e^{i \hat{\phi}(x)}\right\},
$$

and the corresponding density operator

$$
\hat{\rho}(x)=\left[\rho_{0}-\frac{1}{\pi} \partial_{x} \hat{\theta}(x)\right] \sum_{m=-\infty}^{+\infty} e^{2 i m\left(\hat{\theta}(x)-\pi \rho_{0} x\right)} .
$$

In the following, we shall first consider the longwavelength approximation, i.e. $\lambda_{c} \gg 1 / \rho_{0}$, where we can only keep the $m=0$ term in Eqs. (2) and (31). In this limit, the system can be expressed by the hydrodynamical description, with the weak dynamical periodic potential coupled to the slow part of the density operator $\hat{\rho}(x)$. Then, the corresponding low-energy effective Hamiltonian of the atomic part reads

$$
\begin{aligned}
\hat{H}_{a}^{\prime}= & \int_{0}^{L} d x\left\{\frac{\hbar v_{s}}{2 \pi}\left[K\left(\partial_{x} \hat{\phi}(x)\right)^{2}+\frac{1}{K}\left(\partial_{x} \hat{\theta}(x)-\pi \rho_{0}\right)^{2}\right]\right. \\
& \left.-\frac{\hat{V}(x)}{\pi} \partial_{x} \hat{\theta}(x)\right\} .
\end{aligned}
$$

Here, $K$ is the dimensionless parameter and $v_{s}$ the sound velocity. They both depend on a single dimensionless interacting parameter $\gamma=M g_{1 d} / \hbar^{2} \rho_{0}$, with $v_{s} K \equiv v_{F}=$ $\hbar \pi \rho_{0} / M$ fixed by Galilean invariance. We note that, the Hamiltonian (44) describes a LL coupled to a weak periodic potential, which is dynamically dependent on the atomic state and determined self-consistently.

We further transform the Hamiltonian (4) to momentum representation and then implement the standard bosonization procedure by introducing the bosonic creation operator $\hat{b}_{q}^{\dagger}=\sqrt{\frac{2 \pi}{|q| L}} \hat{\rho}_{q}$. We can arrive at the following effective Hamiltonian of the coupled system

$$
\begin{aligned}
\hat{H}_{\mathrm{eff}}= & \sum_{q= \pm 2 k} \hbar \omega_{q} \hat{b}_{q}^{\dagger} \hat{b}_{q}+\hbar g \sum_{q= \pm 2 k}\left(\hat{b}_{q}^{\dagger}+\hat{b}_{q}\right) \hat{c}^{\dagger} \hat{c}+\hbar \Delta \hat{c}^{\dagger} \hat{c} \\
& +i \hbar \eta\left(\hat{c}^{\dagger}-\hat{c}\right) .
\end{aligned}
$$

Here the first term describes the long-wavelength densityfluctuations of the 1D interacting gas with $\omega_{q}=v_{s}|q|$ for $|q| \ll \rho_{0}^{-1}$. The second term is the coupling between the corresponding density-fluctuations and cavity field with $g=\frac{U_{0}}{4} \sqrt{\frac{k L}{\pi}}$. In the sums, we assume that only the $q=$ $\pm 2 k$ modes are coupled to the cavity, which is justified by the low photon numbers limit. $\Delta=\omega_{c}-\omega_{p}+U_{0} N / 2$ is the effective cavity detuning.

The above effective Hamiltonian (5) actually describes the optomechanical coupling between a mechanical oscillator (with the frequency $\omega_{M}=\omega_{ \pm 2 k}=2 k v_{s}$ ) and the radiation pressure force of a cavity field. To see this, we introduce the quadratures of the bosonic excitations $\hat{X}_{M}=\sum_{q= \pm 2 k}\left(\hat{b}_{q}^{\dagger}+\hat{b}_{q}\right) / \sqrt{2}$, and derive the following Heisenberg-Langevin equations

$$
\begin{aligned}
& \frac{d^{2} \hat{X}_{M}}{d t^{2}}+\omega_{M}^{2} \hat{X}_{M}=-2 \sqrt{2} g \omega_{M} \hat{c}^{\dagger} \hat{c}, \\
& \frac{d \hat{c}}{d t}=-i \Delta_{\text {eff }} \hat{c}+\eta-\kappa \hat{c}+\sqrt{2 \kappa} \hat{c}_{\mathrm{in}},
\end{aligned}
$$

with the resonance frequency $\Delta_{\text {eff }}=\Delta+\sqrt{2} g \hat{X}_{M}$ and $\sqrt{2 \kappa} \hat{c}_{\text {in }}$ the noise term. Here, the low-energy longwavelength density fluctuation (phonon) plays the role of a mechanical resonator.

Now, we investigate the optomechanical coupling governed by the set of coupled Eqs. (66)-(7). One of the characteristic phenomenon of cavity optomechanics is the bistable behavior of the stationary solutions, which we derive as $\bar{X}_{M}=-2 \sqrt{2} g|\bar{c}|^{2} / \omega_{M}$, and $|\bar{c}|^{2}=\eta^{2} /\left[\kappa^{2}+\right.$ $\left.\left(\Delta-4 g^{2} \omega_{M}^{-1}|\bar{c}|^{2}\right)^{2}\right]$. In Fig. 2 2 , we give the bistability for three typical oscillator frequency $\omega_{M}$, which correspond to different quantum phases of the system (see below). The linear stability analysis shows that the middle branch (dashed line) is unstable, while the up and low branches are stable. Here, the typical experimental parameters are used: $L \sim 100 \mu \mathrm{m}, \lambda_{c}=780 \mathrm{~nm}, N \simeq 5000$ ${ }^{87} \mathrm{Rb}$ atoms, with $1 / \rho_{0} \simeq 20 \mathrm{~nm} \ll \lambda_{c}$ satisfing the wavelength approximation; $\kappa=2 \pi \times 1 \mathrm{MHz}, U_{0}=2 \pi \times 20$ $\mathrm{kHz}$ with the Rabi frequency $g_{0}=2 \pi \times 10.9 \mathrm{MHz}$ and the pump-atom detuning $\omega_{p}-\omega_{a}=2 \pi \times 32 \mathrm{GHz}$. 

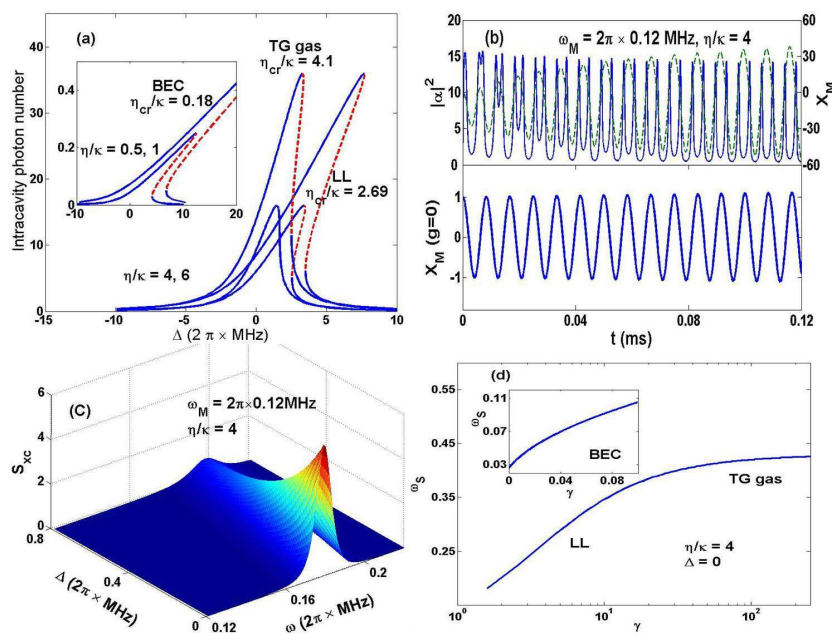

FIG. 2: (color online). Steady-state and dynamical behavior of the optomechanical coupling. (a) Mean cavity photon number versus $\Delta$ for $\omega_{M}=2 \pi \times$ [0.02 (BEC), 0.12 (LL), 0.28 (TG gas)] MHz. Here $\eta_{\text {cr }}$ is the bistability threshold. (b) Cavity photon number $|\alpha|^{2}$, coupled oscillator $X_{M}$ (dashed line), and free oscillator $X_{M}(g=0)$ for $\Delta=0$. (c) Noise spectrum $S_{\mathrm{X}_{\mathrm{c}}}$ versus $\omega$ and $\Delta$. (d) Center frequency $\omega_{S}$ of $S_{\mathrm{X}_{\mathrm{c}}}$ versus $\gamma$. Inset shows the results for small $\gamma$ region.

To discuss the dynamics of the optomechanics, we have taken into account the lossy and driven cavity, where quantum jumps in the cavity photon number can lead to a strong entanglement between the cavity photon number and bosonic wavefunction. This creates a displacement noise spectrum of the mechanical oscillator $S_{X_{M}}(\omega)=$ $2 \kappa\left(4 g \bar{c} \omega_{M}\right)^{2}\left[\kappa^{2}+(\tilde{\Delta}+\omega)^{2}\right] /|d(\omega)|^{2}$, and the corresponding measurable noise spectrum of the cavity field quadrature $\hat{X}_{c}=\left(\hat{c}^{\dagger}+\hat{c}\right) / \sqrt{2}$ 24], which gives $S_{X_{c}}(\omega)=$ $\left[(2 g \bar{c} \tilde{\Delta})^{2} S_{X_{M}}(\omega)+2 \kappa\left[\kappa^{2}+(\tilde{\Delta}+\omega)^{2}\right]\right] /|d(\omega)|^{2}$ (see Fig. 2 c). Here, $d(\omega)=\left(\omega^{2}-\omega_{M}^{2}\right)\left[(\kappa-i \omega)^{2}+\tilde{\Delta}^{2}\right]+2 \omega_{M} \tilde{\Delta}(2 g \bar{c})^{2}$, and $\tilde{\Delta}=\Delta-(2 g \bar{c})^{2} / \omega_{M}$.

We then explore the interatomic interactions dependence of the effective optomechanics. First we note that, whether the system is in weakly or strongly interacting regions, one can realize the effective mechanical oscillator in the whole regions. This implies that the 1D bosonic gas is in fact in a universal class, which can be well described by the low-energy hydrodynamical theory. However, the mechanical oscillator frequency $\omega_{M}$ is fully dependent on interatomic interaction parameter $\gamma$ with $\omega_{M}=2 k v_{s}$. Here, the sound velocity is given by $v_{s}=\sqrt{\left(\rho_{0} / M\right) \partial^{2} E / \partial N^{2}}$, and $E=\sum_{l} \hbar^{2} k_{l}^{2} / 2 M$ is the ground-state energy of $1 \mathrm{D}$ bosonic gas with $k_{l}$ determined by the following Bethe-Ansatz equations [14]

$$
k_{l} L=2 \pi I_{l}-\sum_{m=1}^{N} \tan ^{-1}\left(\frac{k_{l}-k_{m}}{\gamma \rho_{0}}\right),
$$

where $I_{l} \in\{-(N-1) / 2, \cdots,(N-1) / 2\}$ are the set of integers. We solved Eq. (8), and in Fig. 3 we show the numerical results of $\omega_{M}$ versus $\gamma$. Experimentally, when photons enter the cavity, the light filed and the bosonic excitations are coupled nonlinearly, the eigenfrequency of Eqs. (6)-(7) will modify $\omega_{M}$. Nevertheless, the optomechanical coupling is fully determined by the frequency $\omega_{M}$ of bosonic excitations and thereby dependent on $\gamma$ of the 1D gas. Accordingly, by measuring on the cavity field oscillations or detecting the noise spectrum, one can determine the continuous BEC-TG gas crossover, which is an intriguing result of the system.

In the weakly interacting region, we can use the Bogoliubov approximation to derive $\omega_{M}=$ $2 k v_{F} \sqrt{\gamma-\gamma^{3 / 2} /(2 \pi)} / \pi$ for $\gamma \leq 10$ (the dashed line of Fig. 3). However, we note that $\omega_{M}$ vanishes as $\gamma \rightarrow 0$, which contradicts with recent experiments [8]. In fact, as we know because the bosons begin to (quasi) condense for $\gamma \ll 1$, the dominant contribution of the density fluctuation $\hat{\rho}_{ \pm 2 k}=\hat{\Psi}_{ \pm 2 k}^{\dagger} \hat{\Psi}_{q=0}+\sum_{q \neq 0} \hat{\Psi}_{ \pm 2 k+q}^{\dagger} \hat{\Psi}_{q}$ is the quasi-particle excitations from macroscopic occupied $q=0$ ground state. The energy of a quasiparticle is then determined by the Bogoliubov excitation spectrum $\omega_{M}=\sqrt{\epsilon_{ \pm 2 k}\left(\epsilon_{ \pm 2 k}+2 g_{1 d} \rho_{0}\right)} / \hbar$ (the dotted line of Fig. 3). In this case, the mechanical oscillators are the sidemode excitations of a BEC; and in the limit of $\gamma=0$ we get the bare oscillator frequency $\omega_{M}^{0}=4 \hbar k^{2} / M$ (inset of Fig (3). Accordingly, the coupling between the oscillator and a cavity field should be replaced by $g=U_{0} / 2 \sqrt{N / 2}$ in Eqs. (6)-(7), which is enhanced by the condensation. We give the stationary bistability in the inset of Fig. 2a and then numerically integrate the coupled equations for $\Delta=0$ by switching on $\eta / \kappa=4$ at $t=0$. We find that the cavity field oscillates regularly (not shown), but the frequency of the cavity field has a large shift of $\omega_{M}$, which agrees well with Ref. [8].

When $\gamma$ is further increased above unity, the collective density excitations become dominant and the 1D gas crosses to a LL [17. Numerical integration of Eqs. (6)(7) shows that both $|\alpha|^{2}$ and $X_{M}$ exhibit well-defined oscillations, and the cavity field is excited resonantly at $X_{M}=0$ (Fig. 2b). Yet we note that, different from the BEC phase where $g$ is collectively enhanced, the optomechanical coupling becomes small. In this case, the linear stability analysis shows that the eigenfrequency of the set of coupled equations is nearly the same with the free oscillator $\omega_{M}$, see Fig. 20 for example. This is a characteristic phenomenon of the region. Experimentally, by increasing the interatomic interactions, if the oscillation frequency of the cavity field follows the the solid line of Fig. 3, the 1D gas should be in the LL phase. We also calculate the noise spectrum $S_{\mathrm{X}_{\mathrm{c}}}$ (Fig. 25), where the center frequency $\omega_{S}$ of the spectrum has a shift of $\omega_{M}$. In Fig. 2d, we give $\omega_{S}$ for $\Delta=0$ in the whole interacting regions. We find that $\omega_{S}$ increases with $\gamma$ and has a tendency to saturate above $\gamma \simeq 50$, which can be inspected in experiments.

While for $\gamma \gg 1$, the strong interactions would 


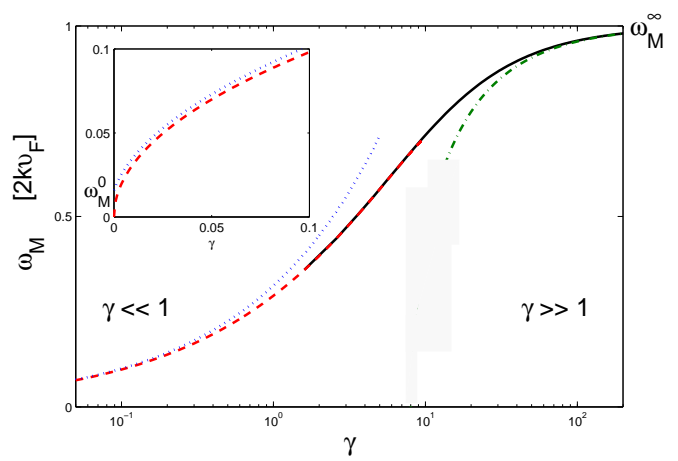

FIG. 3: (color online). Mechanical oscillator frequency $\omega_{M}$ versus $\gamma$. The solid line is the numerical Bethe-Ansatz result. The dashed and the dash-dotted lines are the asymptotic results in the weakly and strongly interacting regions respectively. In the weakly interacting region, we also give the Bogoliubov excitation spectrum (the dotted line).

prevent the bosons from occupying the same position. Especially when $\gamma=\infty$, the symmetric many-body wave function of bosons can be mapped to an antisymmetric fermionic wave function by $\Psi_{B}\left(x_{1}, \cdots, x_{N}\right)=$ $A\left(x_{1}, \cdots, x_{N}\right) \Psi_{F}\left(x_{1}, \cdots, x_{N}\right)$ with $A\left(x_{1}, \cdots, x_{N}\right)=$ $\prod_{1 \leq j<k \leq N} \operatorname{sgn}\left(x_{k}-x_{j}\right)$ [13]. Hence, the Hamiltonian (1) can be rewritten in terms of the fermion field operators

$$
\hat{H}_{F}=\frac{\hbar^{2}}{2 M} \int_{0}^{L} d x \partial_{x} \hat{\Psi}_{F}^{\dagger}(x) \partial_{x} \hat{\Psi}_{F}(x)+\int_{0}^{L} d x \hat{V}(x) \hat{\rho}_{F}(x),(9)
$$

which is exactly the model describing a free fermion gas subjecting to the cavity periodic potential [25]. Then, the oscillator is formed by the particle-hole excitations at the edges of $\pm k_{F}$ through the Bose-Fermi mapping, and the mechanical frequency is naturally related to the Fermi velocity: $\omega_{M}^{\infty}=2 k v_{F}=2 k \hbar \pi \rho_{0} / M$. While for finite $\gamma$, we use the asymptotic expression to derive $\omega_{M}=2 k v_{F}[1-4 / \gamma]$ (the dash-dotted line of Fig. 3). Experimentally, when the 1D gas becomes a TG gas, the oscillation frequency of the cavity field should follow this asymptotic expression and approaches $\omega_{M}^{\infty}$. Correspondingly, the center frequency $\omega_{S}$ will saturate at $2 \pi \times 0.42$ $\mathrm{MHz}$ (Fig. 2d).

Finally, let us turn to the commensurate situation with $\lambda_{c} \sim 2 / \rho_{0}$, where a new instability-the sine-Gordon transition-may appears in the strongly interacting $1 \mathrm{D}$ quantum gas [19]: the superfluid ground state turns insulating in the presence of a weak commensurate periodic potential. It is now necessary to take account of the discrete nature of boson with $m \neq 0$ terms in Eq. (3). This gives rise to a sine-Gordon type perturbation [18] up to the leading term

$$
\hat{H}_{\mathrm{s}-\mathrm{G}}=\frac{1}{2} \hbar U_{0} \hat{c}^{\dagger} \hat{c} \rho_{0} \int_{0}^{L} d x \cos [2 \hat{\theta}(x)+Q x],
$$

where $Q=2 \pi\left(\rho_{0}-k / \pi\right)$, which vanishes at commensurability. In the small photon numbers limit, it was shown that [26], this term is renormalization irrelevant for $K>K_{c}=2$ or equivalently $\gamma<\gamma_{c}=3.5$, leaving the ground state a superfluid LL with the same linear excitation spectrum as the long-wavelength approximation. Then, one expects a well-defined oscillation of optomechanical coupling. While for $\gamma>\gamma_{c}, \hat{H}_{\mathrm{S}-\mathrm{G}}$ becomes relevant, the system transits to an insulating Mott phase, where the bosonic excitations are forbidden owing to the energy cost, and the optomechanical oscillation vanishes correspondingly. Therefore, we can use the optomechanical dynamics across the critical point $\gamma_{c}$ to detect the sine-Gordon transition easily in experiments.

In summary, we demonstrate that one can realize the effective optomechanics in the whole interacting regions of $1 \mathrm{D}$ bosonic gas. This offers a new approach to to detect the BEC-TG gas crossover or the sine-Gordon transition by investigating the optomechanical coupling. These proposals are of particular significance for exploring novel phenomena of cavity QED and ultracold atoms.

This work is supported by NSFC under grants No. 11704175, the NKBRSFC under grants No. 2011CB921500. X. C. Xie is supported in part by USDOE under Grant No. DE-FG02-04ER46124.

[1] F. Brennecke et al., Nature (London) 450, 268 (2007).

[2] Y. Colombe et al., Nature (London) 450, 272 (2007).

[3] K. Baumann et al., Nature (London) 464, 1301 (2010).

[4] D. Nagy et al., Phys. Rev. Lett. 104, 130401 (2010).

[5] J. Keeling et al., Phys. Rev. Lett. 105, 043001 (2010).

[6] S. Gupta et al., Phys. Rev. Lett. 99, 213601 (2007).

[7] K. W. Murch et al., Nat. Phys. 4, 561 (2008).

[8] F. Brennecke et al., Science 322, 235 (2008).

[9] For a review of recent advances of optomechanics, see T. Kippenberg and K. Vahala, Science 321, 1172 (2008).

[10] V. B. Braginsky et al., Science 209, 547 (1980).

[11] D. S. Goldbaum et al., arXiv:0911.3234v1.

[12] A. Görlitz et al., Phys. Rev. Lett. 87, 130402 (2001).

[13] M. Girardeau, J. Math. Phys. (N.Y.) 1, 516 (1960).

[14] E. H. Lieb and W. Liniger, Phys. Rev. 130, 1605 (1963); E. H. Lieb, Phys. Rev. 130, 1616 (1963).

[15] B. Paredes et al., Nature 429, 277 (2004).

[16] T. Kinoshita et al., Science 305, 1125 (2004).

[17] H. Moritz et al., Phys. Rev. Lett. 91, 250402 (2003).

[18] H. P. Büchler et al., Phys. Rev. Lett. 90, 130401 (2003).

[19] E. Haller et al., Nature 466, 597 (2010).

[20] We note that, all the methods to determine a BEC [12], TG gas [15, 16], BEC to TG gas crossover [17], or the sine-Gordon transition [19] of the 1D interacting bosonic gas are destructive.

[21] I. B. Mekhov et al., Nat. Phys. 3, 319 (2007).

[22] C. Maschler et al., Phys. Rev. Lett. 95, 260401 (2005).

[23] F. D. M. Haldane, Phys. Rev. Lett. 47, 1840 (1981).

[24] M. Paternostro et al., New J. Phys. 8, 107 (2006).

[25] R. Kanamoto et al., Phys. Rev. Lett. 104, 063601 (2010).

[26] A. O. Gogolin, A. A. Neresyan, and A. M. Tsvelik, Bosonization and Strongly Correlated Systems, (Cambridge University Press, Cambridge, 1998). 